\title{
A repeat audit of the Cognitive Analytic Therapy Clinic at Guy's Hospital
}

\author{
Mark Dunn, Katya Golynkina, Anthony Ryle and J. P. Watson
}

\begin{abstract}
A repeat audit of reforrats to the CAT Clinic of Guy's Hoepltal is reported. Changes in training and superviston of theraplats instlfuted aince earlier audits are belleved to have contributed to a lower drop-out rate and a higher attendance for follow-up. Mean score reductions on three questionnaires are significant and consistent in the three cohorts. Some characteristics of samples of patients who dropped out and falled to attend for follow up are described.
\end{abstract}

Cognitive Analytic Therapy (CAT) has been available at Guy's for 12 years. It originated as a training exercise for hospital staff of various professional backgrounds, but grew rapidly as requests for training from non-hospital professionals provided an increasing number of trainee therapists.

By 1993, the year audited in the present report, the CAT clinic was run by one whole time equivalent of administrative and clerical staff. The work of more than 30 trainees, most of whom treated four cases in the course of the year, was supervised by one whole time equivalent supervisor. No paid or fully trained therapists were available.

\section{The treatment method}

CAT was developed with the aim of providing psychotherapy within the NHS: it is delivered in a 16 session format in the great majority of cases. It integrates theory and practice ideas from cognitive-behavioural and psychoanalytic approaches. Its main features are the active participation of the patient and the early written and diagrammatic reformulation of the patients' problems, as described by Ryle (1990, 1995).

Supervision and training were initially carried out by AR, who was primarily responsible for the psychotherapy service at St Thomas's Hospital, which was similarly dependent on trainees. At both sites supervision was given free in exchange for the therapy provided. Trainees came with varying levels of prior counselling or psychotherapy experience. From the more experienced, a group of CAT supervisors and teachers emerged who went on to form what is now the national Association for Cognitive Analytic Therapists (ACAT). These developments were accompanied by a steady increase in the academic input offered to trainees. In 1992 an advanced course resulting in a UKCP recognised psychotherapy training admitted its first trainees and the first 15 graduates qualified in 1994-95.

\section{The scope of CAT}

Experience with a wide range of patients suggests that CAT is a safe first intervention for nonpsychotic patients provided they are not seriously substance abusing; the intake criteria are therefore broad. Patients with untreated depression are normally referred for pharmacological treatment before receiving therapy. Patients with borderline personality disorder are accepted and their treatment is currently being evaluated; these patients receive up to 24 sessions.

\section{Previous audit}

The first audit concerned patients seen between February 1988 and August 1990. Towards the end of this period a comparative audit of the Guy's and St Thomas's services was carried out (Denman, 1995). This drew attention to the many deficiencies in the data collection methods then available, and also indicated a high attrition rate among patients at both hospitals. At Guy's, 23\% of patients who had been booked in for therapy falled to attend their first therapy session and a further $33 \%$ failed to complete therapy. In a separate audit of the quality of therapy provided. based upon audio tapes of sample sessions, Denman (1993) reported widely differing levels of skills shown in theraples carried out by trainee therapists and proposed a number of remedies.

\section{Changes since the last audit}

Partly as a result of these audits and partly as a result of the general evolution of better training methods in CAT, a number of changes have been 
instituted. Data collection has been improved by the provision of a clerical worker able to collect data and maintain the database, and by the development of effective methods for getting adequate levels of cooperation from the large number of trainee therapists. Clinical standards have been improved by providing more training and support for supervisors, by the reduction in size of supervision groups from 5 to 4 or less, and by an increase in the training input for trainees. One other change was instituted: during the 1988-90 period patients referred by psychiatrists were accepted without a CAT psychotherapy assessment. This practice was stopped and a formal assessment introduced as it was felt that both selection of suitable cases and an adequate explanation of the nature of the therapy were difficult to provide in psychiatric out-patient interviews.

\section{Audit of the 1993 cohort}

The present audit can demonstrate how far the changes summarised above have been effective, as well as offering measures against which the service can be compared with other NHS psychotherapy resources which might offer comparable information. The aims of this audit were:

(1) To record 1993 demographic and referral data

(2) to compare attrition rates and outcome of treated patients with previous audits

(3) to seek to identify the nature of patients who did not complete therapy and of those who failed to attend for follow-up.

\section{Method}

Demographic and referral data and progress through the system are now automatically recorded on the database. Outcome is measured by comparing pre- and post-therapy scores on four questionnaires: Social Questionnaire (SQ; Corney et al, 1982), Beck Depression Inventory (BDI; Beck et al, 1961; Beck \& Steer, 1987), the Symptom Check List (SCL-90-R; Derogatis, 1977) and the Inventory of Interpersonal Problems (IIP; Horowitz et al, 1988). In addition, a post-therapy semi-structured assessment interview was carried out at which individual problems and problem procedures identified in the early sessions of therapy were rated for change. detailed examples of any reported change being sought. On this basis the assessor and patient rate 'target problem' and 'target problem procedure' change on a 5-point scale $(1-$ much improved, 5-much worse), the least improved rating (usually the assessor's) being used.
Samples of patients who dropped out of treatment and of those completing but failing to attend for follow-up were studied and compared with those completing and attending.

\section{Results}

The sample

In the group of referred patients there were more women than men ( $58 \%$ female); $70 \%$ of patients were under the age of 40; the level of educational attainment was relatively high $(86 \%$ reported GCSE or ' $O$ ' level or higher); $60 \%$ were single or divorced; $46 \%$ reported belonging to either Catholic or Protestant faith: $10 \%$ were homosexual; $14 \%$ belonged to ethnic minorities.

The most frequent clinical diagnosis was minor depression and anxiety (58\%); $37 \%$ had a history of parasuicide; $12 \%$ were heavy drinkers.

Of 243 referrals, 145 came from hospital psychiatrists, 27 GPs and 71 from other community resources.

Attrition: (a) before and (b) after the first therapy session compared to previous audits

(a) Between 1992 and 1993, referrals increased from 158 to 243 . There were no significant differences in the proportion going on to treatment (70\% and $67 \%$ respectively). Of those who did not go on to treatment in the 1993 cohort, 53 patients did not respond to initial contact, 21 were referred back at assessment as inappropriate and five did not turn up for session 1.

(b) An increasing proportion of patients completed treatment $(62 \%$ in $1990,76 \%$ in 1992 and $82 \%$ in 1993). Also an increasing proportion of patients managed to attend follow-up $(30 \%$ in $1990,49 \%$ in 1992 and $52 \%$ in 1993).

\section{Outcome}

Psychometric data based on those completing and attending follow-up.

Table 1 records the pre-therapy and posttherapy scores on the four measures used: Social Questionnaire, Beck Depression, the Symptom Check List-90-R and the Inventory of Interpersonal Problems for the 1993 cohort, based on patients who attended for follow-up assessment at between 3 and 6 months post-therapy.

SQ scores show no significant change but on other inventories significant reductions in mean scores are achieved. This is consistent across the 3 years of the audit. The percentage of patients on the various categories according to BDI preand post-therapy scores are presented in Flg. 1. 
Table 1. Pre- and post-therapy mean psychometric scores on four questionnaires

\begin{tabular}{lllll}
\hline & \multicolumn{1}{c}{ SQ } & \multicolumn{1}{c}{ BDI } & SCL-90-R & IIP \\
\hline $\begin{array}{l}\text { Pre-therapy } \\
\text { mean (s.d.) }\end{array}$ & $20.93(10.29)$ & $21.89(10.42)$ & $1.44(0.69)$ & $1.7(0.63)$ \\
Post-therapy & $19.04(9.55)$ & $13.56(9.92)$ & $0.9(0.7)$ & $1.3(0.6)$ \\
$\quad$ mean (s.d.) & 1.68 & 6.66 & 6.62 & 6.41 \\
t-test & 0.096 & $<0.001^{*}$ & $<0.001^{*}$ & $<0.001^{*}$ \\
$P$ & & & & \\
\hline
\end{tabular}

*Significant values

Changes in pre- and post-therapy scores on the questionnaires did not differ significantly from those in 1988-90 and 1992.

Mean ratings for target problems at the followup assessment meeting were 1.8 (s.d. $=0.64$ ), and for target problem procedures 1.98 (s.d. $=0.72$ ).

Of the 135 patients who completed therapy, 25 (18.5\%) were referred for other treatment; including group therapy, sex/couple therapy, more CAT, longer-term therapy and OP psychiatric support.

Characteristics of patients not completing therapy and failing to attend for follow-up In order to identify the distinguishing characteristics of unsuccessful cases, samples of the following two groups of patients were studied: (1) patients starting but not completing therapy and (2) patients who complete therapy but fail to attend for follow-up (FFU). These samples were selected before the data on the whole 1993 sample had been collected but are otherwise random.

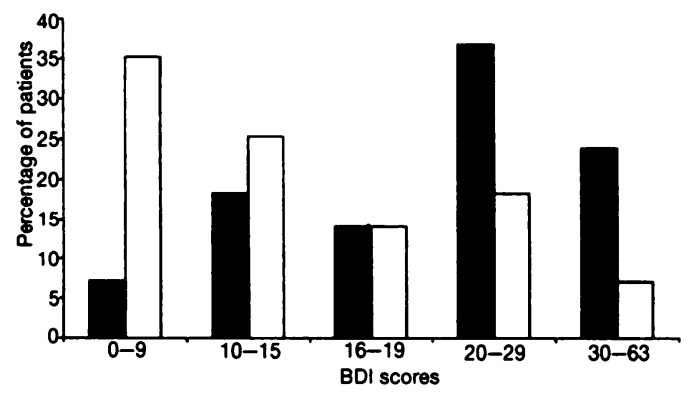

BDI scores: 0-9, normal; 10-15, mild depression; 16-19. mild to moderate; 20-29, moderate to severe; $30-63$, severe depression.

Fig. 1. The percentage of patients on the various categories according to BDI scores $(n=71)$. Pre-therapy BDI scores; $\square$, Post-therapy BDI scores
Characteristics of patients not completing therapy: This study was based on a sample of 21 of the 28 non-completers. Non-completers were found to have significantly higher initial scores on the BDI (Mean score 29.05; s.d. $=11.62$ ) and were more likely to have a history of substance abuse.

Characteristics of those who fail follow-up: The failure to attend for follow-up could reflect a range of attitudes, including a wish to move on after a difficult time, or resentment at an unsuccessful intervention. Against the latter explanation is the fact that decisions about offering further treatment are made after the follow-up assessment, which might make attendance a sign of continuing need.

Pre-therapy inventory scores of a sample of 18 of the $\mathbf{4 9}$ failed follow-up patients were compared with a sample of 67 completers. There were no significant differences in the mean scores, indicating that failure to attend cannot be predicted by psychometric testing.

The records of the therapies of the failed followup sample were inspected. On this basis 13 (68\%) patients in this group were classified as probably improved, three (16\%) as probably not improved and in three $(16 \%)$ cases there was insufficient evidence.

\section{Discussion}

A comparison of the 1993 audit with those from earlier years shows that the referral rate has increased but that the psychometric characteristics of the referred patients have not altered. In each of the three cohorts studied, mean scores on the BDI, SCL-90 and IIP fell significantly in those attending the follow-up. The increasing percentage of patients who, having attended the first session, complete therapy, and the increasing proportion of those who attended for followup, suggest that the changes implemented in training and supervision after the previous audits have had a positive effect.

Although an uncontrolled study, the fact that only $18.5 \%$ of patients are referred on for further treatment suggests a satisfactory impact for an 
intervention of 16 sessions carried out by trainees. This figure might be higher were more resources available. A more detailed understanding of the factors influencing outcome will require research into process and outcome in more closely defined patient groups; work of this sort is currently underway in patients with borderline personality disorder (see Ryle \& Marlow, 1995; Bennett \& Parry, 1997).

Psychotherapy services have not published clinical audits of this type so far, but the study by Amies (1996) of his patient population records slightly higher levels of disturbance compared with those recorded here.

The data presented here will serve as a basis for comparison with future cohorts and with the work of other clinics offering either CAT or other models of intervention. In the current climate the demonstration of the effectiveness of treatments may influence funding decisions. For this purpose psychometric measures are relatively simple to apply once a database is established and a clerical worker provided to maintain it. While measures of this sort do not address the full range of changes sought in psychotherapy, patients who are judged at the post-therapy assessment to need further treatment because of persistence of limiting or damaging procedures have significantly higher psychometric scores.

While this audit shows improved adherence to treatment and satisfactory outcomes in most cases, the attrition between referral and starting therapy has slightly increased. The main reason for this is probably the continuing long delays between assessment and commencing therapy (currently around six months).

Some changes in the audit procedures are proposed. In future it is intended to collect psychometric data: (a) on referral; (b) when there is a delay of more than three months before starting; (c) mid-therapy; (d) at termination, and (e) at follow-up. This may give further information concerning those who fail to complete therapy, those who do not improve and those who fail to attend for follow-up, and might indicate that a flexdble contract for the length of therapy, depending on early progress, might be appropriate. It is also proposed to attempt to identify more systematically those patients meeting the diagnostic criteria for borderline, socio- pathic and narcissistic personality disorders, as these patients present greater difficulties in treatment.

\section{References}

AMIES, P. (1996) Psychotherapy patients: are they the worried well'? Psychiatric Bulletin, 20, 153-156.

BECK, A. T., WARD, C. H., MENDELSON, M., et al (1961) An inventory for measuring depression. Archlves of General Psychiatry, 4, 561-571.

- \& STEER. R. A. (1987) Manual for Revised Beck Depression Inventory. New York: Psychological Corporation.

BENNETT, D. \& PARRY, G. (1997) The accuracy of reformulation in cognitive-analytic therapy: a comparison of two methods for the identification of recurrent relationship patterns. Psychotherapy Research (in press).

CORney, R. H., Clare, A. W. \& Fry, J. (1982) The development of a self-report questionnaire to Identify social problems - a pilot study. Psychological Medictne. 12. 903-909.

DENMAN. F. (1993) Quality in a psychotherapy service: a review of audiotapes of sessions. Psychiatric Bulletin. 18. 80-82.

- (1995) Auditing CAT. In Cognitive Analytic Therapy: Developments in Theory and Practice (ed. A. Ryle). Chichester: John Wiley.

DEROGATIS, L. R. (1977) SCL-90-R: Administration, Scoring and Procedures Manual-II. Towson. MD: Clinical Psychometric Research.

HOROWITZ, L. M., ROSENBERG, S. E., BAER, B. A., et al (1988) Inventory of interpersonal problems: psychometric properties and clinical applications. Journal of Consulting and Clinical Psychology. 66, 885-892.

Rne. A. (1990) Cognittue Analytic Therapy: Active Participation in Change. Chichester: John Wiley.

- (1995) Cognitue Analytic Therapy: Developments in Theory and Practice. Chichester: John Wiley.

- \& MARLOWE, M. J. (1995) Cognittve analytic therapy for borderline personality disorder: theory and practice and the clinical and research uses of the self states sequential diagram. International Journal of Short Term Psychotherapy. 10, 21-34.

Mark Dunn, Psychotherapist; "Katya Golynkina, Research Fellow: Anthony Ryle, Hon. Consultant Psychotherapist and Senior Research Fellow; and J. P. Watson, Professor, Division of Psychiatry and Psychology, UMDS, Guy's Hospital, London Bridge, London SE1 9RT

*Correspondence 